\title{
Correction of Single Frequency Altimeter Measurements for Ionosphere Delay
}

\author{
William S. Schreiner, Robert E. Markin, and George H. Born
}

\begin{abstract}
This study is a preliminary analysis of the accuracy of various ionosphere models to correct single frequency altimeter height measurements for ionospheric path delay. In particular, research focused on adjusting empirical and parameterized ionosphere models in the parameterized real-time ionospheric specification model (PRISM) 1.2 using total electron content (TEC) data from the global positioning system (GPS). The types of GPS data used to adjust PRISM included GPS line-of-sight (LOS) TEC data mapped to the vertical, and a grid of GPS derived TEC data in a sun-fixed longitude frame. The adjusted PRISM TEC values, as well as predictions by IRI-90, a climatological model, were compared to TOPEX/Poseidon (T/P) TEC measurements from the dual-frequency altimeter for a number of T/P tracks. When adjusted with GPS LOS data, the PRISM empirical model predicted TEC over $241 \mathrm{~h}$ data sets for a given local time to within a global error of $8.60 \mathrm{TECU}$ rms during a midnight centered ionosphere and 9.74 TECU rms during a noon centered ionosphere. Using GPS derived sun-fixed TEC data, the PRISM parameterized model predicted TEC within an error of 8.47 TECU rms centered at midnight and 12.83 TECU rms centered at noon. From these best results, it is clear that the proposed requirement of $3-4$ TECU global rms for TOPEX/Poseidon Follow-On will be very difficult to meet, even with a substantial increase in the number of GPS ground stations, with any realizable combination of the aforementioned models or data assimilation schemes.
\end{abstract}

\section{INTRODUCTION}

A ATELLITE altimetry has become a very powerful tool $\$$ for the study of ocean circulation and variability and provides data for understanding important issues related to climate and global change. Sea surface height measurements are computed by combining the radar altimeter measurement with knowledge of the orbit height of the satellite. Thus, any errors in the altimeter and orbit height measurements map directly into the sea surface height observables and reduce the ability to extract the desired ocean signal from the data. One of the many error sources in the altimetry process is the delay in the altimeter measurement caused by the charged particles in the earth's ionosphere. For a $13.6 \mathrm{GHz}$ altimeter, a total electron content (TEC) of 1 TECU $\left(10^{16} 16\right.$ electrons $/ \mathrm{m}^{2}$ ) corresponds to approximately $0.218 \mathrm{~cm}$ of range delay. A maximum expected TEC (at solar maximum or during solar storms) of $10^{18} 18$ electrons $/ \mathrm{m}^{2}$ will create $22 \mathrm{~cm}$ of

\footnotetext{
Manuscirpt received April 20, 1995; revised March 1, 1996. This work was supported by NASA Grant Nag-1-1491 under the direction of Steve Katzberg of the NASA Langley Research Center.

W S Schreiner is with the University Corporation for Atmospheric Research, University of Colorado, Boulder, CO 80301 USA.

R. E Makin and G. Born are with the Colorado Center for Astrodynamics Research, University of Colorado, Boulder, CO 80309 USA.

Publisher Item Identifier S 0196-2892(97)00371-9.
}

range delay. Since some ocean signals have centimeter level magnitudes, it is necessary to correct for ionosphere delay in the altimeter measurements. If a radar altimeter transmits at two frequencies, a first-order linear combination of the two signals can calibrate the delay to a sufficient level. However, because several missions, including Geosat follow-on (GFO), to be launched in late 1997, and the ongoing European Space Agency's ERS-1 and ERS-2 use of single frequency altimeters, calibration of ionosphere delay is a subject of considerable interest.

This study was undertaken to investigate techniques with the potential of supplying a measure of the sub-satellite TEC for the purpose of correcting altimeter range measurements. The T/P follow-on (TPFO) mission requires TEC measurements accurate to $2.5-4 \mathrm{TECU}(0.5-0.8 \mathrm{~cm}$ range correction), based on the performance of T/P [1]. Reference [2] defines accuracy as the root mean square difference between the measurement and "truth" value for a large sample. For this study, we adopt the T/P TEC measurements as "truth."

Because climatological (monthly mean) models are known to be in error by as much as $50 \%$, this work has focused on the parameterized real-time ionospheric specification model (PRISM), capable of improving its TEC prediction by ingesting (adjusting to) in situ ionospheric measurements. Two types of data derived from the dual frequency L band GPS signals were used to adjust PRISM: GPS satellite to ground station data mapped from the line-of-sight (LOS) to the vertical at the point of intersection of the LOS with the ionosphere shell model at $350 \mathrm{~km}$ altitude. The second is a grid map of GPS-derived TEC data in a sun-fixed longitude frame [3].

Another ionosphere TEC source available is the Doppler Orbitography and Radiopositioning Integrated by Satellite (DORIS) data provided on the T/P geophysical data records. The DORIS data are based on LOS TEC between the host satellite, T/P, to the ground stations. It is dependent on localized coverage by $\mathrm{T} / \mathrm{P}$ and does not contain mesoscale information. Furthermore, the data from DORIS will be for a fixed time of day depending on the location of the line of nodes of the host satellite. This time of day will vary as the node regresses; however, the data will only be of significant value to an altimeter flying in the same time of day orbit. Although correcting TEC using DORIS data should be examined, other data and models currently available provide more globally useful range correction data.

Because GPS is the only measurement system to offer global observations of the ionosphere, this research has focused on evaluating the PRISM model, using global GPS TEC 
data as input, by comparing PRISM TEC predictions to T/P dual-frequency TEC measurements (considered as truth). Additionally, the sun-fixed GPS TEC grids and the climatological international reference ionosphere (IRI-90) were investigated as independent TEC predictors. Thus, the primary objectives were to:

1) Determine if adjusting the PRISM model with global GPS TEC (both mapped vertical TEC and the TEC grid map) data results in sub-satellite TEC predictions that are accurate to within an instantaneous error of $4 \mathrm{TECU}$, and if the method cannot supply the required accuracy, to determine the reason and the accuracy that can be expected.

2) Investigate other techniques and data sets for improving the PRISM model prediction.

3) Evaluate and compare to other aforementioned models and methods for predicting TEC.

\section{PRISM}

PRISM was developed for the United States Air Force (USAF) Air Weather Service by Computational Physics, Inc. The goal of the model is to provide a near real-time specification of the ionosphere over the entire globe. PRISM predicts the composition of the ionosphere using two models, an URSI model, which is a set of interpolation coefficients for empirical estimation of the ionosphere, and a physical model, which is based on parameterized physical models of the various layers of the ionosphere. The parameterized model divides the ionosphere into four separate physical layers and uses both ground-based and satellite-based measurements of the ionosphere to adjust physical parameters to more accurately determine ionospheric composition. This adjustment procedure can correct eight profile parameters at the data locations, using a weighting function, dependent on distance of the point of interest from the ingested data point, to specify a global ionosphere correction field [4]. For single frequency altimeter calibrations, the goal is to ingest third-party ionospheric data into PRISM to more closely predict actual sub-satellite TEC.

PRISM [4] employs a procedure which enables it to adjust the parameterized physical model using a variety of ionosphere data. These data types include: bottomside soundings of the digital ionosphere sounding system, TEC data from any source, and in situ plasma and auroral electron and ion fluxes from the DMSP satellites. Before any real-time adjustment is made, PRISM uses linear interpolation on $F_{10.7}$ and $K_{p}$ to obtain the best prediction of the state of the ionosphere from the parameterized data bases. Once this is obtained, the realtime adjustment procedure uses the available data to correct for eight profile parameters at each data site. In between each measurement site, as will often be the case for the altimeter application, a weighted average based on distance is used to interpolate the eight adjustment parameters. Fig. 1 shows the original weight function used in the PRISM adjustment procedure. This function helps to ensure that PRISM matches the data at each measurement site and that TEC will vary smoothly between sites. The large drop off of this function exists such that information relatively far (greater than $500 \mathrm{~km}$ ) from a

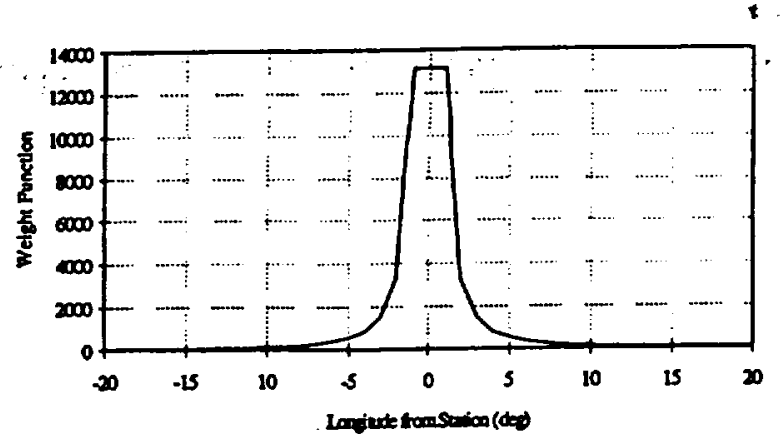

Fig. 1. Original PRISM weight function versus longitude at the equator. This function essentially de-weights data more than $3^{\circ}$ from the measurement site.

site will not be used. This function, however, is somewhat deceiving in that if the point of interest is far from any data source, a weighted average of all data will be performed, and in such a case, TEC data from any site will influence predictions of TEC at distances much greater than $500 \mathrm{~km}$ [5].

\section{IRI-90}

The international reference ionosphere (IRI-90) model, developed by the Committee on Space Research and the International Union of Radio Science, is an extensively researched climatological model. IRI-90 describes monthly averages of electron density and temperature as well as ion temperature and composition in the altitude range from $50 \mathrm{~km}$ to $1000 \mathrm{~km}$ for magnetically quiet conditions in the nonauroral ionosphere [6]. The model is based on empirical data from ionosonde measurements, incoherent scatter observations, rocket ion mass spectrometers and various other data sets, as well as analytic functions developed to fill in the gaps. Combining several techniques and algorithms to interpret the data, IRI-90 generates interpolation equation coefficients for determining ionospheric composition from the atmospheric measurements to describe monthly mean vertical profiles for the main parameters of the ionosphere [6], [7].

\section{GLOBAL GPS TEC DATA}

Deriving GPS TEC data that is suitable for input into the PRISM model consists of measurements from the two $\mathrm{L}$ band signals $(\mathrm{Ll}$ at $1575.42 \mathrm{MHz}$ and $\mathrm{L} 2$ at $1227.6 \mathrm{MHz}$ ) that, in theory, can be linearly combined in a straightforward manner to compute a measure of the TEC between the GPS satellite and receiver. In practice, however, this computation is complicated by the presence of hardware biases between the $\mathrm{L} 1$ and L2 channels in both the GPS satellite and GPS receiver. To derive an absolute measure of LOS TEC, these biases must be solved for (or calibrated if possible) and removed from the data. This absolute LOS TEC must then be mapped to an equivalent vertical TEC for ingesting by PRISM. The GPS network used in this study, shown in Fig. 2, is the 33 station network that was available in March of 1993.

The first step in the procedure to generate absolute vertical TEC data is to form the biased LOS TEC data from the raw dual frequency measurements. A biased measure of TEC can be computed from the dual frequency pseudorange data. By performing a least squares fit (leveling) of the carrier 


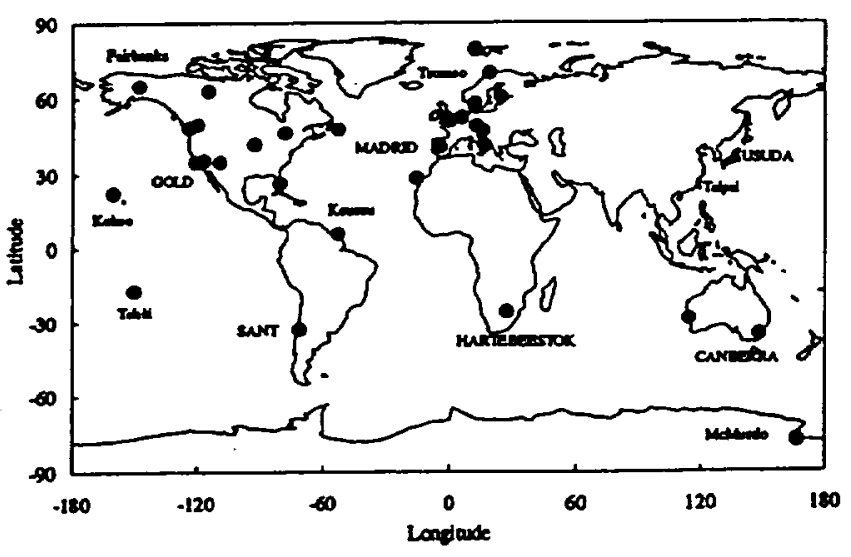

Fig. 2. GPS tracking network, 33 station configuration available in March 1993.

phase signal TEC data to the pseudorange TEC data over a given orbit pass, a precise LOS TEC measurement biased only by the receiver and satellite hardware biases (and not carrier cycle ambiguities) can be generated. This is given by $\mathrm{TEC}_{\text {mess }}=\mathrm{TEC}_{\text {true }}+b_{\text {sat }}+b_{\mathrm{rcvr}}$, where $b_{\text {sat }}$ and $b_{\text {rcvr }}$ are the satellite and receiver hardware biases, respectively.

The next step is to remove the $\mathrm{L} 1 / \mathrm{L} 2$ hardware biases, $b_{\text {sat }}$ and $b_{\text {revr }}$ which are either estimated as constants along with the grid TEC values or taken from the receiver hardware calibration. These biases can then be subtracted from the TEC measurements to obtain absolute LOS TEC from the GPS satellite to the receiver [3]. Once these measurements have been formed, they can be mapped to the vertical at the intersection of the measurement and the shell using an infinitely thin ionosphere shell assumption [8]. Thus, for a given receiver and at given time, there will be a number of - Ttical TEC measurements that have been mapped to varying s) b-ionospheric latitude and longitude intersection points.

The uncertainties in the derived vertical GPS TEC data are composed of both random and systematic effects attributed to measurement noise in the least squares fits between the pseudorange and carrier phase data and uncertainties in the $\mathrm{L} 1$ and $\mathrm{L} 2$ hardware biases. The maximum expected vertical GPS TEC data uncertainties can be obtained [9] by dividing the LOS uncertainties by a mapping function [8] to give maximum uncertainties in the receiver hardware biases of $0.76 \mathrm{TECU}$ rms when calibrating and 1.14 TECU $\mathrm{ms}$ when estimating. The uncertainties do not include errors due to the vertical mapping process. The worst case maximum vertical uncertainties could contribute to problems for meeting the 4 TECU TPFO requirement.

\section{GRID MAPS}

Using tracking data from the GPS network, a group at the Jet Propulsion Laboratory (JPL) has developed a means of processing data from ground based GPS receivers to generate a 642 point global hourly grid of vertical TEC (and uncertainty) in a sun-fixed longitude reference frame [3], [10], [11]. This is accomplished by taking mapped vertical GPS TEC data over a $24 \mathrm{~h}$ period and rotating in longitude to the sun-fixed frame. (Zero hour sun-fixed longitude has been defined as 12 h GMT for Greenwich longitude.) This data is then processed to give estimates of the TEC associated with each grid point. Additionally, the process estimates GPS satellite and receiver biases as constants, which can be used in estimating uncertainties in the TEC data as previously described and for calculating absolute TEC from GPS data.

The grid consists of a network of stochastic (random walk) points in time that are updated hourly, along with their covariances, as new GPS TEC data are acquired. If GPS TEC data are not present over a grid point, the estimate of the grid point is not updated at that time, and its uncertainty increases according to the noise assigned to the stochastic parameter. The TEC and covariances at each grid point are interpolated to a one-by-one degree resolution map to give estimates globally at every longitude and latitude point. Because the model was developed in a sun-fixed frame as a function of time, near global coverage can be attained. The accuracy is not as much limited by the spatial decorrelation of the ionosphere as it is by the temporal correlations (over a few hours) and the coverage and distribution of the GPS receivers.

\section{TOPEX DUal Frequency Data}

The T/P geophysical data records contain all relevant altimetric data including dual-frequency ionospheric range correction data which can be converted to LOS TEC. Measurements from the $T / P$ altimeter consist of round trip light times of both the $\mathrm{Ku}$ and $\mathrm{C}$ band signals (13.6 and $5.3 \mathrm{GHz}$ ) off the ocean surface. In theory, these measurements can be used directly to compute the TEC between the altimeter and the ocean surface, but in truth are compted by a hardware bias between the $\mathrm{Ku}$ and $\mathrm{C}$ band channels. The $\mathrm{Ku}$ and $\mathrm{C}$ band relative offsets were estimated (at about $1.7 \mathrm{~cm}$, or an 8 TECU effect) by the T/P project at JPL using histograms of the ionosphere TEC data [12] allowing for an accurate determination of TEC. Besides accounting for channel biases, other corrections that are applied to the T/P TEC data include estimates of pointing angle errors and varying $K u$ and $C$ band sea state (i.e., electromagnetic bias) effects.

The uncertainties in the derived T/P TEC data are comprised of both random and systematic effects. The random effect is due to noise in the $\mathrm{Ku}$ and $\mathrm{C}$ band range measurements. Smoothed over $20 \mathrm{~s}$, the error due to this noise is approximately $2 \mathrm{~mm}$, or 1 TECU [2]. The systematic errors are more difficult to quantify, although it is believed that the $10 \mathrm{~cm}$ relative $\mathrm{Ku}$ and $\mathrm{C}$ band offset is accurate to approximately $2 \mathrm{~cm}$ [12] corresponding to about $1.8 \mathrm{TECU}$ error $(0.4 \mathrm{~cm}$ at $\mathrm{Ku}$ band). Ignoring the error caused by the differing band electromagnetic biases, an estimate of the uncertainty of the T/P TEC data can be computed by taking the root sum square of the measurement noise and the uncertainty of the relative $\mathrm{Ku}$ and $\mathrm{C}$ band offset giving a value of $2.1 \mathrm{TECU}$, much smaller than the worst case GPS TEC data uncertainty $(5.5$ TECU) [9].

\section{RESULTS}

A set of globally distributed TEC measurements were generated using GPS data (courtesy JPL) from March 12 , 


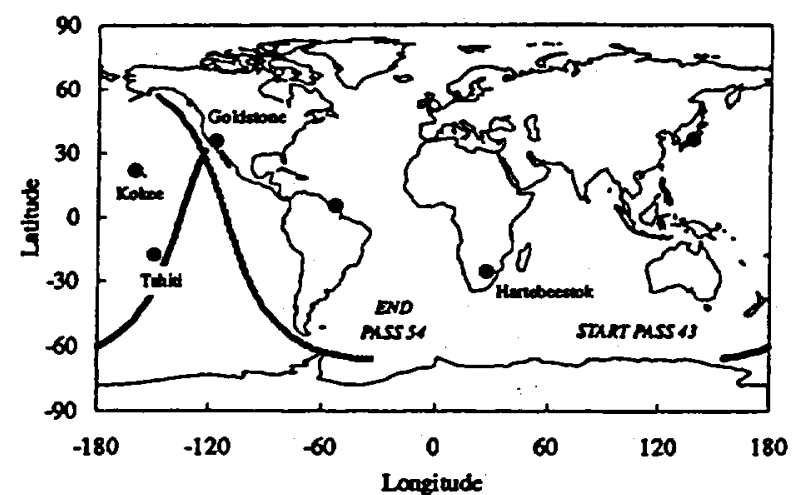

Fig. 3. TOPEX groundtracks for passes 43 and 54 (cycle 18) with nearby GPS stations (black dots).

1993, for input into PRISM. Post-processed estimates of solar and geophysical data were obtained from the National Geophysical Data Center in Boulder, Colorado, to allow the PRISM unadjusted base model to be as accurate as possible. March 12 was a moderately active day with an $F_{10.7}$ and sun spot number of 158.7 and 77.0, respectively. The GPS configuration used for this study was the 33 station network available during March 1993 (see Fig. 2).

The PRISM parameterized model was used to generate TEC for a number of T/P sub-satellite tracks in cycle 18 for this day. The PRISM adjusted (with only raw vertical mapped GPS TEC data) and unadjusted values were compared with the TOPEX TEC data at one minute intervals. The TOPEX 1 $s$ TEC data was smoothed over $20 \mathrm{~s}$ centered on $1 \mathrm{~min}$ intervals [1] and were examined along T/P groundtracks. Fig. 3 shows groundtracks and the relative geometry for TOPEX passes 43 and 54 with the closest GPS stations. Local times at the midpoint of each pass are approximately 1 am for ascending pass 43 and noon for descending pass 54. Johnson et al, [5] demonstrate in their primary analysis that the ionosphere is most active and variable around local noon, and the agreement between PRISM predictions and TOPEX truth is worse in the early afternoon than any at other time of day. This being the case, the best test of adjusting PRISM to match TOPEX TEC would necessarily reside in predicting a local noon-time ionosphere. Night-time ionospheres were also examined for gaining additional information regarding the technique and procedure.

Fig. 4 gives TOPEX and PRISM TEC for pass 43 , showing little improvement to PRISM when ingesting raw GPS TEC data, yielding identical differences, 5.3 TECU $\mathrm{ms}$, from TOPEX TEC data for the adjusted and unadjusted predictions. Though not reflected by this rms, it is obvious that the PRISM model is affected by the GPS TEC data as noted by the jump in the PRISM adjusted values between 31260 and 31380 s (i.e., sixth and eighth data points) as a result of PRISM switching from a high-latitude ionosphere model to a midlatitude procedure which uses ingested data differently. In addition, the Tahiti GPS station improves PRISM values near the point of closest approach at $32100 \mathrm{~s}$, but offers little improvement when far away at $32420 \mathrm{~s}$. There is also a near overflight of a California station, but the TEC for this

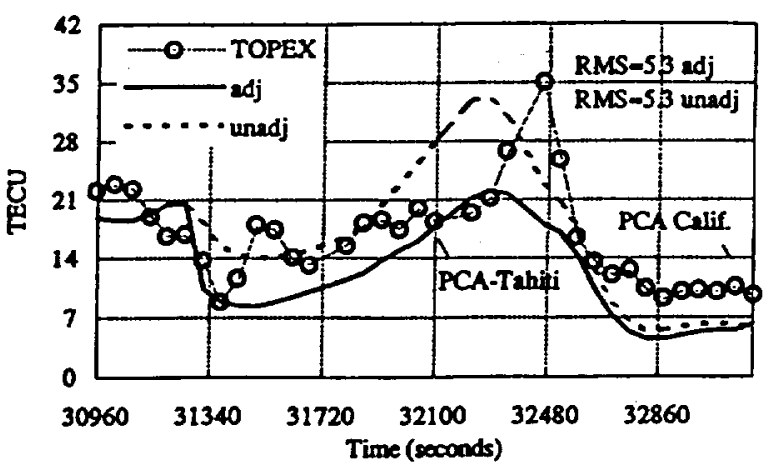

Fig. 4. TEC from PRISM unadjusted and adjusted with raw GPS TEC data compared with TOPEX TEC for March 12, 1993, for pass 43 in cycle 18.

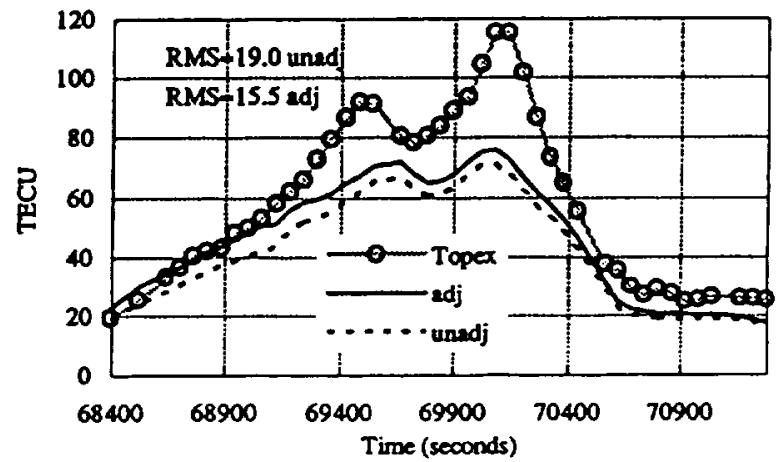

Fig. 5. TEC from PRISM unadjusted and adjusted with raw GPS TEC data compared with TOPEX TEC for March 12, 1993, for pass 54 in cycle 18.

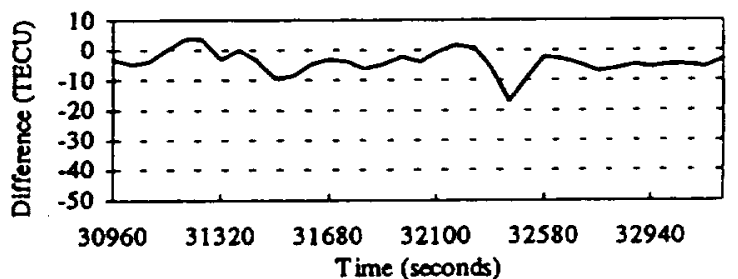

Fig. 6. Peak errors between TOPEX TEC and predictions by PRISM for pass 43 (local time $\sim 1 \mathrm{am}$ ).

night-time ionosphere is too small to notice any significant adjustment.

Fig. 5 shows TEC data for pass 54 , which traverses a daytime ionosphere near local noon exhibiting maximum effects near 120 TECU as seen by T/P. Slight improvements are seen in the PRISM TEC values when using GPS TEC data, but the adjusted rms difference, 15.5 TECU, is still well above the desired accuracy requirement with peak data excursions as great as $40 \mathrm{TECU}(8 \mathrm{~cm}, \mathrm{Ku}$ band). Note that for the night-time pass 43 , the peak errors are less than $20 \mathrm{TEC}$ in magnitude (see Figs. 6 and 7). Again, this is due to a lack of stations in the vicinity of the pass when it enters the maximum ionosphere. Another investigation for predicting daytime ionosphere (pass 52 , not shown) showed improvement from 12.7 TECU rms with no additional adjustment as compared with $8.50 \mathrm{TECU}$ rms adjusted, where the prediction was aided by an overflight of the Santiago GPS station near midday. 


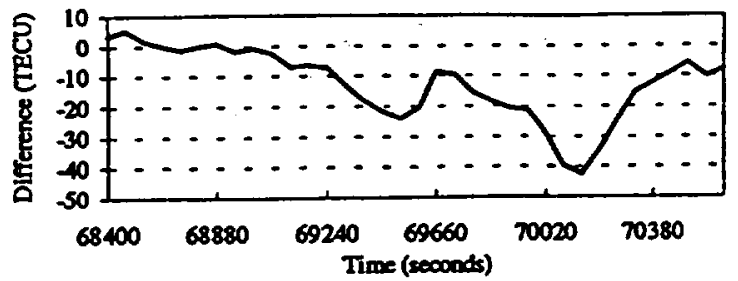

Fig. 7. Peak errors between TOPEX TEC and predictions by PRISM for pass 54 (local time noon).

These results demonstrate that the PRISM adjustment procedure matches the T/P TEC values reasonably well when the groundtrack passes near a GPS station. This is because the PRISM weight function used for adjusting incorporates information from a TEC measurement within 300 or $400 \mathrm{~km}$ of that measurement. For this reason, the weight function was modified to incorporate information up to $1000 \mathrm{~km}$ away from the measurement, having understood that this may result in some decorrelated information being used. PRISM was run again over the same three passes using the modified weight function giving rms differences of 7.1, 6.9, and 14.4 TECU, for passes 43,52 and 54 , respectively, compared with original weight function rms differences of 5.3, 8.5, and 15.5 TECU. Passes 52 and 54 yielded only modest improvement, while pass 43 results actually degraded. These results are inconclusive and show only that using a generalized weight function with a large decorrelation distance does not necessarily yield better results.

At this point, the weighting function used by PRISM was modified specifically for ingesting the JPL grid TEC data such that the grid data would be weighted based on expected accuracy, or uncertainty, at each grid point instead of merely on distance from a known measurement. This was accomplished by changing the equation of the exponential in the weighting function to be additionally dependent on the variance of the grid data. The amount of dependence was then optimized by minimizing the rms differences between the PRISM TEC predictions and the TOPEX truth TEC over a $24 \mathrm{~h}$ period.

Both the unadjusted parameterized and empirical URSI PRISM models were used to generate TEC values for comparison with T/P dual-frequency TEC measurements for all sub-satellite tracks in cycle 18 for March 13, 1993 (the reason for the change in day of interest was to make use of the available JPL TEC grid data centered on March 13). The model predictions over this day's period were then adjusted separately with raw GPS TEC data using the original weighting function and adjusted with sun-fixed TEC grid maps using the weighting function optimized for that data type. These were compared to TOPEX TEC and TEC predictions by the climatological model, IRI-90, and by the sun-fixed maps alone. Graphical results presented here are for pass 60 , a relatively active data set in which straight forward comparisons could be made.

Fig. 8 shows the predictions of the PRISM URSI model unadjusted, adjusted with the weighted JPL grid and optimized weighting function, and adjusted with raw vertical GPS TEC data using the original weighting function. The TOPEX truth

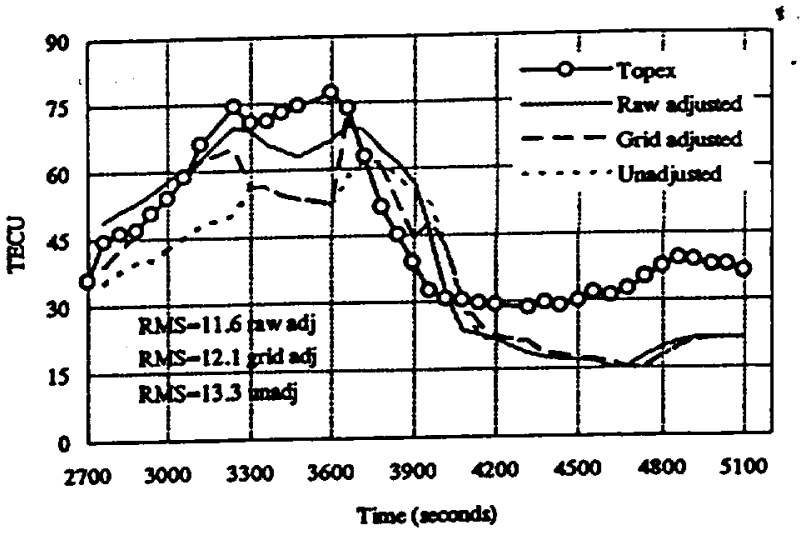

Fig. 8. Smoothed TEC comparisons of PRISM URSI model with TOPEX TEC, cycle 18, pass 60 , (midpoint local time $\sim$ noon).

TABLE I

COMparisons wTth TOPEX TEC, 13 March 1993, CYCLE 18. PAss 60 (MmPPont LOCAL TME NOON), IN TECU

\begin{tabular}{|l|c|c|}
\hline \multicolumn{1}{|c|}{ Model } & mean & Ims \\
\hline PRISM, ursi, JPL GPS grid & -8.15 & 12.09 \\
\hline PRISM, ursi unadjusted & -10.22 & 13.33 \\
\hline PRISM, ursi, vectical GPS (raw) & -5.22 & 11.56 \\
\hline PRISM, par, JPL GPS grid & -8.08 & 12.31 \\
\hline PRISM, par unadjusted & -11.56 & 14.15 \\
\hline PRISM, par, vertical GPS (raw) & -10.98 & 14.23 \\
\hline IPL GPS grid & -5.01 & 13.89 \\
\hline IRI-90 & 4.74 & 11.55 \\
\hline
\end{tabular}

data are also shown. PRISM URSI adjusted with the raw GPS data visibly performed the best. The model adjusted with grid data began well but developed significant errors when the uncertainties in the grid became unreasonably large between 3240 and $3480 \mathrm{~s}$ causing the model to revert back to the base model. Table I gives rms and mean differences between TOPEX TEC and model predictions.

Fig. 9 gives the predictions of the PRISM parameterized model for the same three cases of adjustment. For this PRISM base, the model adjusted with the weighted grid data conformed best to the general structure and data of the TOPEX TEC. The rms errors and mean deviations of the parameterized model predictions also are given in Table I. From these errors, it is obvious that during a daytime ionosphere none of the methods predict TEC very accurately. The figures and data indicate that all of these models underpredict the TOPEX TEC, and some even have difficulty in modeling the general structure of the ionosphere. It should also be noted that, although IRI90 performs quite well for this specific TOPEX pass, when a larger data set is examined, the overall performance of this model is somewhat degraded (i.e., see Table II).

Over a $24 \mathrm{~h}$ data set sampling local times of approximately noon and 1 am (i.e., passes 60 through 84), the rms values were computed for each of the base and adjusted models are given in Table II along with combined average values over the $24 \mathrm{~h}$ data set. From this information, it is clear that the PRISM URSI model adjusted with raw vertical mapped GPS TEC was the most accurate in terms of mean and rms TECU differenced with TOPEX data, and is the only model that predicts with an accuracy less than even 10 TECU ms error 
TABLE II

Comparisons to topex TEC, 13 March 1993, Cycle 18, Passes 60-84; Ascending (Odd Numbered. Nighttime), Descendang (Even Numbered, Noontume), and 24 H Data Sampling Combinang all Passes

\begin{tabular}{|c|c|c|c|c|c|c|}
\hline Method & $\begin{array}{c}\text { mean } \\
\text { ascending }\end{array}$ & $\begin{array}{c}\text { Ins } \\
\text { ascending }\end{array}$ & $\begin{array}{c}\text { mean } \\
\text { descending }\end{array}$ & $\begin{array}{c}\text { mos } \\
\text { descending }\end{array}$ & $\begin{array}{c}\text { mean } \\
\text { combinod }\end{array}$ & $\begin{array}{c}\text { mins } \\
\text { combined }\end{array}$ \\
\hline PRISM, ursi unadjusted & -7.22 & 12.47 & -10.44 & 14.11 & -8.8 & 13.3 \\
\hline PRISM, par. unadjusted & .2 .19 & 8.79 & -9.00 & 14.71 & -5.6 & 12.1 \\
\hline PRISM, wri, GPS raw & -2.22 & 8.60 & -3.96 & 9.74 & -3.1 & 92 \\
\hline PRISM, par, GPS raw & -3.64 & 8.33 & -8.23 & 14.00 & -5.9 & 115 \\
\hline PRISM, ursi, JPL grid & -5.93 & 10.87 & 8.73 & 12.69 & -7.3 & 11.8 \\
\hline PRISM, par. JPL grid & -3.63 & 8.47 & -7.57 & 12.83 & -5.6 & 10.9 \\
\hline IRL-90 & 5.86 & 11.19 & 5.23 & 13.16 & 5.5 & 12.2 \\
\hline
\end{tabular}

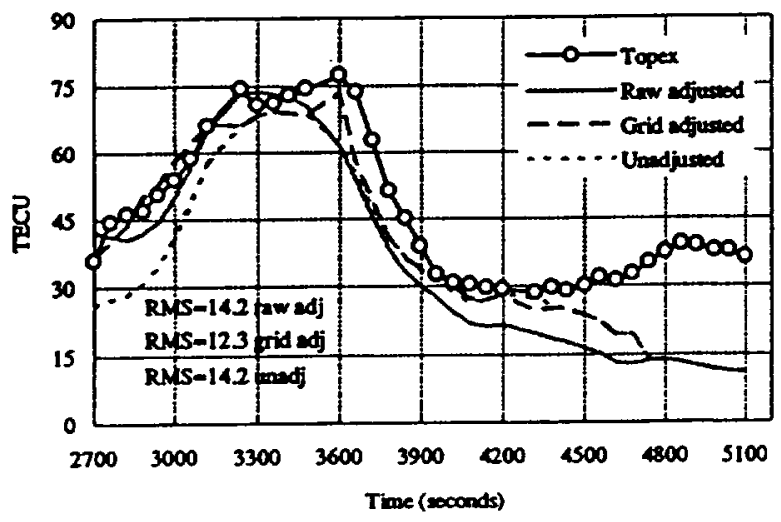

Fig. 9. Smoothed TEC comparisons of parameterized PRISM with TOPEX TEC, cycle 18, pass 60 , (midpoint local time, $\sim$ noon).

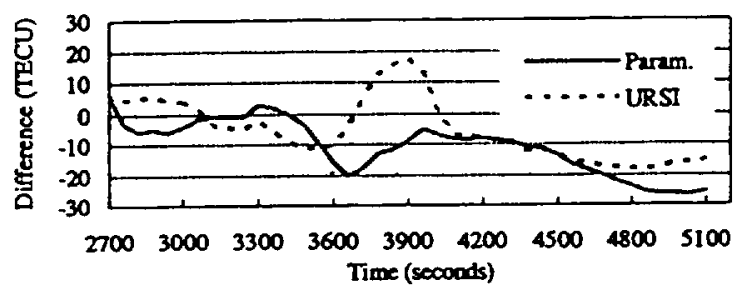

Fig. 10. Peak errors between T/P TEC and predictions by PRISM URSI and parameterized models (pass 60).

during a daytime ionosphere, still far from meeting a 4 TECU requirement. These predictions were differenced from TOPEX TEC measurements at each latitude (and corresponding time) over every available pass in the day, and near 20 TECU peak differences ( 30 for the parameterized model) were commonly seen, translating into $3-4 \mathrm{~cm}$ errors in sea surface height (Fig. 10). Although the actual TEC predictions from the JPL grid are given, the fact that the GPS coverage exhibits huge uncertainties over large areas lends little reliability to the grid as a stand-alone TEC predictor. This would be remedied by a denser net of ground stations.

One note regarding PRISM predictions is that the average TECU error for almost every method is negative, indicating that the models consistently under-predict T/P TEC. One contributing factor is a possible bias of as much as $5 \mathrm{TECU}$ found in the TOPEX/Poseidon data by the T/P Project Office [1], [2]. Accounting for this bias would decrease the mean deviation of the model predictions and, to an extent, lower the rms as well.

\section{DISCUSSION OF RESULTS}

The adjusted PRISM values generally matched the TOPEX measurements within 10 TECU rms when the sub-satellite track passed within $300-400 \mathrm{~km}$ of a GPS station or when the track passed through a night time ionosphere. However, when the points of interest were greater than $300-400 \mathrm{~km}$ away from vertically mapped GPS station data or when a local noon ionosphere was sampled, the adjusted PRISM values generally differed by greater than 10 TECU with data excursions from TOPEX TEC of as much as $40 \mathrm{TECU}$ (an $8 \mathrm{~cm}$ path delay error at $\mathrm{Ku}$ band). Using a modified weight function (taking into account information up to $1000 \mathrm{~km}$ away from GPS station data) showed no appreciable improvement in the PRISM results. Therefore, it may be concluded from this analysis that ingesting TEC data from the current (March 1993) set of GPS stations directly into PRISM (version 1.2) will not predict sub-satellite TEC globally within an accuracy of 4 TECU rms, much less instantaneously as indicated by peak errors shown in Figs. 6, 7, and 10. Because the PRISM adjustment incorporates measurement information within $300-400 \mathrm{~km}$ (based on the spatial ionosphere decorrelation distance) of the T/P overflight point, a prohibitively large number of ionospheric measurement sites would be needed to provide input for PRISM in order to consistently meet any accuracy requirement less than $10 \mathrm{TECU}$.

The performance of PRISM using JPL grid data is, however, promising considering this technique has only recently been developed. Marked improvement should be realized when advantage is taken of an expanding GPS network. Use of the sun-fixed TEC grid data for ingestion into PRISM should be studied further, particularly if grid maps based on observables from the now denser GPS network could be employed. In addition, PRISM ingestion of DORIS data would likely yield similar accuracies as did the GPS data and grid maps and should also be examined at some point. However, it is clear that the TPFO requirement of 3-4 TECU global accuracy will be very difficult to meet using any realizable combination of these existing models and data assimilation schemes.

\section{ACKNOWLEDGMENT}

The authors would like to thank $J$. Carson of the USAF Air Weather Service and D. Anderson of the USAF Phillips Laboratory at Hanscom AFB for access to PRISM; R. Daniell, 
B. Whartenby, and L. Brown at Computational Physics, Inc., for supplying PRISM databases, source code and assistance in the operation of PRISM; A. Mannucci of Section 335 at JPL for supplying the GPS TEC data, estimates, and calibrations of the receiver and satellite L1/L2 biases, and the global sunfixed grid TEC data; D. Bilitza of Hughes, STX, for providing IRI-90; and L. Johnson of Science Applications International Corp. for insight toward understanding the PRISM weighting function. The T/P altimeter data was provided by the Physical Oceanography Distributed Active Archive Center at JPL.

\section{REFERENCES}

[1] P. Calahan, JPL, personal communication, Jan/Feb. 1994.

[2] D. Imel, "Evaluation of the TOPEX dual-frequency ionosphere correction," J. Geophys. Res., vol. 99, no. C12, pp. 24895-24906, Dec. 15, 1994.

[3] A. J. Mannucei, B. D. Wilson, and C. D. Edwards, "A new method for monitoring the earth's ionosphere total electron content using the GPS global network," in Proc. ION GPS-93, Salt Lake City, UT, Sept. 1993, pp. 1323-1332.

[4] R. E Daniell, W. G. Whartenby, and L. D. Brown, "Algorithm description for the parameterized real-time ionospheric specification model version 1.2," Computational Physics, Inc., Newton, MA, May 1993.

[5] J. L. Johnson, D. W. Poter. H. R. Anderson, and G. S. E. Lagerloef, "Radar altimetry ionospheric correction study, final repor," SAIC. Bellevue, WA, 1994.

(6) Intemational Reference Ionosphere 1990, D. Bilitza, Ed., National Space Science Data Center, NSSDC 90-22. Greenbelt, MD, Dec. 1990.

[7] D. Biliza, K. Rawer, L. Bossy, and T. Gulyaeva, "International reference ionosphere-Past, present, and future," Adv. Space Res., vol. 13. no. 3, pp. 3-23, 1993.

[8] G. E. Lanyi and $T$. Roth, "A comparison of mapped and measured total ionospheric electron content using global positioning system and beacon sacellite observations," Radio Sci., vol. 23, no. 4, pp. 483-492. July-Aug. 1988.

[9] W. S. Schreiner, G. H. Born, and R. E. Markin. "Ionospheric calibration for single frequency altimeter measurement." Final Report. Boulder, CO. Mar. 1994.

[10] B. D. Wilson, A. J. Mannucci, and C. D. Edwards, "Global ionosphere maps using a global network of GPS receivers," Beacon Satellite Symp., Cambridge, MA, July 1992.

[11] A. Mannucci, B. Wilson, and D. Yuan, "An improved ionospheric correction method for wide-area augmentation systems," in Proc. ION GPS-95, Palm Springs, CA, Sept. 1995, pp. 1199-1208.

[12] P. Calahan. JPL, personal communication, June 1993.

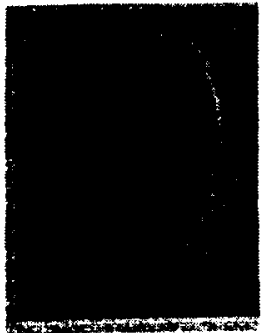

William S. Schreiner received the B.S. degree in seconautical and astronautical engineering from The Obio State University, Columbus, in May 1988 and the Ph.D. degree in aerospace engineering sciences from the University of Colorado, Boulder, in 1993.

From 1989 to 1993 he worked for the Colorado Center for Astrodynamics Research. He is presently a Scientist working for the University Corporation for Atmospheric Research GPS Meteorology (GPSMET) program, which entails processing low earth orbiter and ground-based GPS data for precise orbit determination and radio occultation applications.

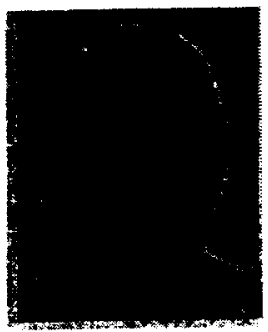

Robert E. Markin received the B.S. degree in aerospace enginecring from the University of Alabama, Huntsville, in 1993, and the M.S. degree in rerospace engincering sciences in 1995 from the University of Colorado, Boulder, where he is currently a Ph.D. student employed by the Colorado Center for Astrodynamics Research. His research includes ionosphere modeling, orbit perturbation theory, and optimal orbit maneuvers.

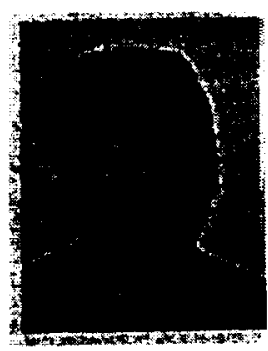

George H. Born received the Ph.D. degree in aerospace engineering from the University of Texas at Austin in 1968.

He was employed at the Manned Spacecrafi Center, Houston, TX, during the Apollo Program. In 1970 he joined the Jet Propulsion Laboratory, Pasadena, CA, here he served as a Technical Group Supervisor for the Mariner 9 and Viking missions to Mars, and the Geophysical Evaluation Manager for the Seasat Project. In 1983 he became a Senior Research Engineer at the University of Texas at Austin. In 1985 he joined the Aerospace Engineering Sciences Department at the University of Colorado. Boulder, as a Professor, and founded the Colorado Center for Astrodynamics Research, which he directs. His research interests are satellite navigation and satellite oceanography, particularly the application of satellite altimetry.

Dr. Born is a fellow of the American Astronautical Society and the American Institute of Aemonautics and Astronautics. 